\title{
CMEARTICLE
}

\section{Electrocardiographic T wave abnormalities}

Weiqin $\underline{\mathrm{Lin}}^{1}$, MBBS, MRCP, Swee Guan $\underline{\operatorname{TeO}}^{2}$, MBBS (Hons), MRCP, Kian Keong $\underline{P o}^{1,3}$, FRCP, FACC

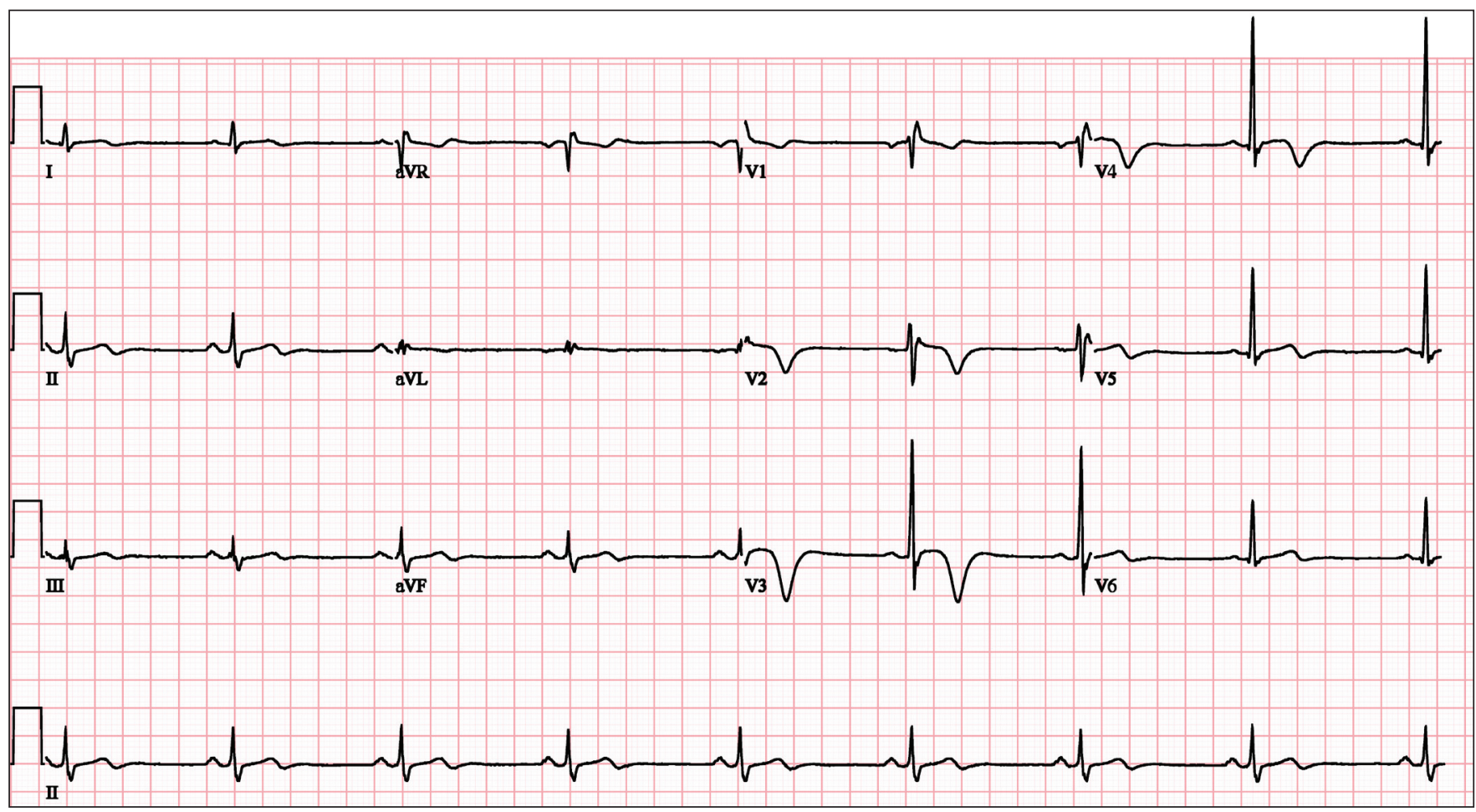

Fig. 1 ECG of patient shows T wave inversions.

\section{CASE 1}

\section{CLINICAL PRESENTATION}

A 61-year-old man was admitted for intermittent chest pain, which lasted for over one week. It worsened on exertion and was associated with diaphoresis. The pain was described as a central squeezing sensation that lasted for about ten minutes and was relieved with rest. Cardiovascular examination of the patient was unremarkable, and he was normotensive at rest. What are the electrocardiographic (ECG) abnormalities seen in Fig. 1?

${ }^{1}$ Cardiac Department, National University Heart Centre, National University Health System, ${ }^{2}$ Raffles Heart Centre, Raffles Hospital, Singapore, ${ }^{3}$ Yong Loo Lin School of Medicine, National University of Singapore, Singapore

Correspondence: A/Prof Poh Kian Keong, Senior Consultant and Associate Professor, Cardiac Department, National University Heart Centre, 1E Kent Ridge Road, NUHS Tower Block, Level 9, Singapore 119228. kian_keong_poh@nuhs.edu.sg 

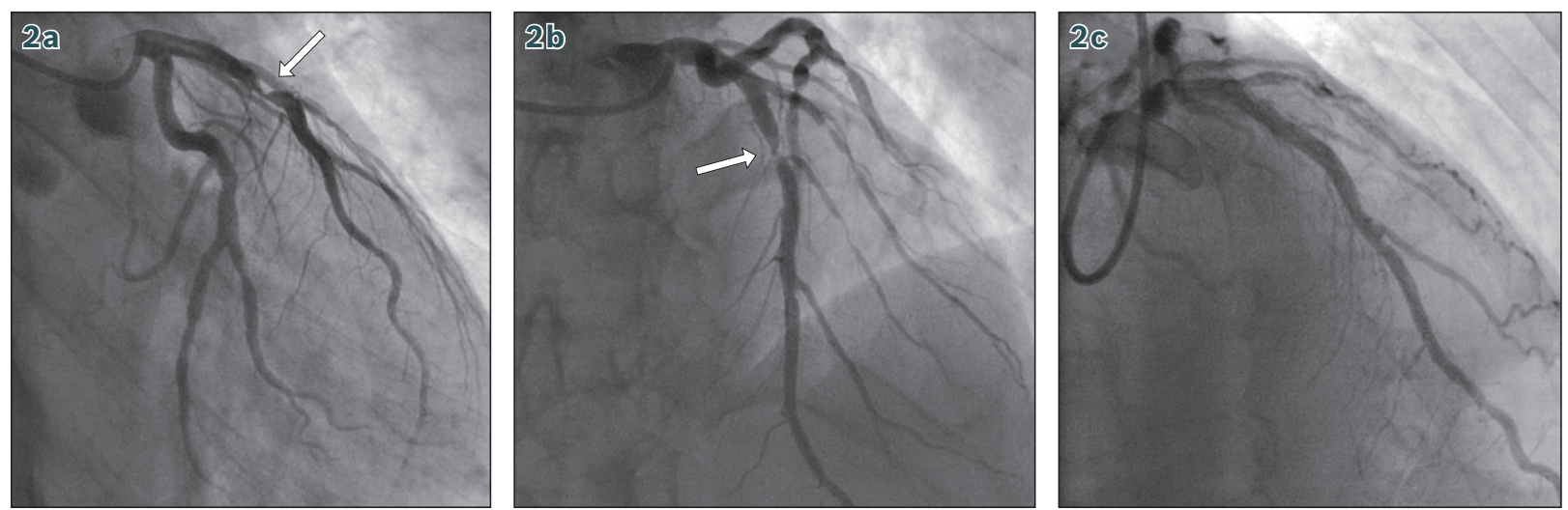

Fig. 2 Coronary angiography in the (a) right anterior oblique caudal and (b) anterior-posterior cranial views of the left coronary artery show $90 \%$ stenosis of the mid segment (arrows). (c) Coronary angiography in the right anterior oblique cranial view of the left coronary artery post angioplasty shows good flow with no residual stenosis.

\section{ECG INTERPRETATION}

The ECG in Fig. 1 shows sinus rhythm with normal PR and QRS durations. There are deep $T$ waves in the chest leads (V2 to V6), as well as biphasic $T$ waves in the inferior leads. There were no ST changes and the ECG findings did not meet the criteria for left ventricular hypertrophy. ${ }^{(1)}$

\section{CLINICAL COURSE}

The patient was treated for ischaemic heart disease. Serial cardiac enzyme studies and ECG examinations performed revealed elevation of cardiac troponins and no change in ECG findings. The patient was then managed for non-ST-elevation myocardial infarction. On coronary angiography, single vessel coronary artery disease with the culprit being a $90 \%$ stenosis of the mid-left anterior descending artery (Figs. 2a \& b) was shown. The patient underwent coronary angioplasty with subsequent stenting of the culprit lesion (Fig. 2c). Subsequent echocardiography showed preserved left ventricular ejection fraction.

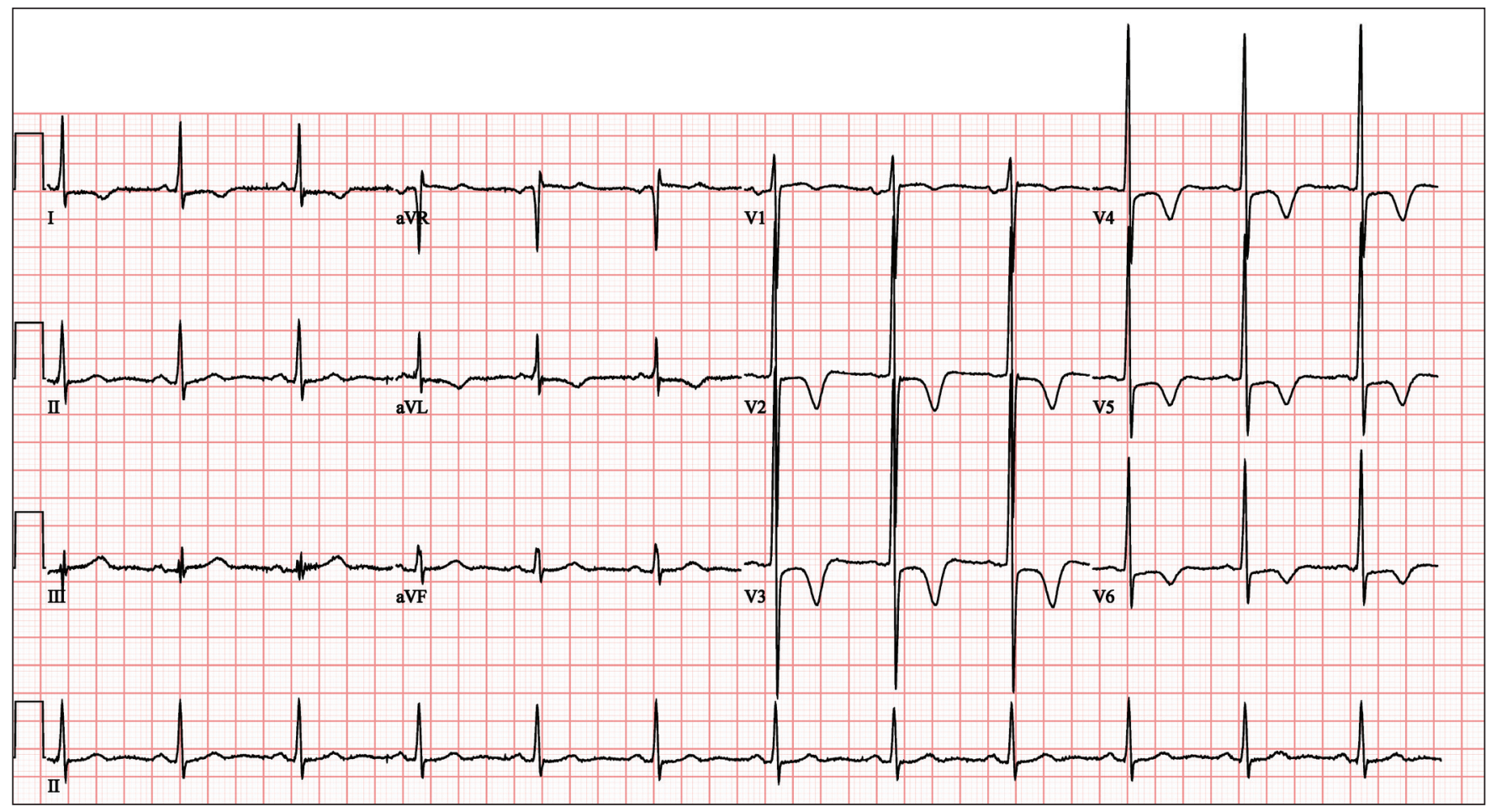

Fig. 3 ECG shows T wave inversions and left ventricular hypertrophy.

\section{CASE 2}

\section{CLINICAL PRESENTATION}

An 80-year-old man presented to the cardiology service for evaluation of a cardiac murmur. He was otherwise asymptomatic, with no exertion chest pain or dyspnoea.
Physical examination revealed a grade 2 ejection systolic murmur, as well as a grade 2 early diastolic murmur at the lower left sternal edge. The patient was normotensive. What does the patient's ECG (Fig. 3) show? 


\section{ECG INTERPRETATION}

The ECG in Fig. 3 shows sinus rhythm in the patient. Both the PR interval and QRS duration were within normal limits. There was also evidence of left ventricular hypertrophy, fulfilling Sokolow-Lyon criteria for left ventricular hypertrophy (i.e. $\mathrm{S}$ wave in $\mathrm{V} 1+\mathrm{R}$ wave in $\mathrm{V} 5$ or $\mathrm{V} 6 \geq 35 \mathrm{~mm}$ ). ${ }^{(2)}$ In this case, the sum was $43 \mathrm{~mm}$. It also fulfilled the Cornell criteria for left ventricular hypertrophy in male patients (i.e. $\mathrm{R}$ wave in $\mathrm{a} \mathrm{VL}+\mathrm{S}$ wave in $\mathrm{V} 3>28 \mathrm{~mm}$ ). ${ }^{(3)}$ There was also widespread $\mathrm{T}$ wave inversion in the anterolateral leads (V2 to V6, I and aVL), as well as downward ST depression in the chest leads (V3 to V6), representing a 'strain pattern'.

\section{CLINICAL COURSE}

Transthoracic echocardiography was performed with an intravenous contrast agent to better delineate the left ventricular borders. Asymmetrical left ventricular hypertrophy at the septum was observed (Fig. 4). However, the gradient across the left ventricular outflow tract was not elevated. These features were consistent with nonobstructive hypertrophic cardiomyopathy. There was also mild aortic, mitral and tricuspid valve regurgitation. The pulmonary artery and aortic root were dilated. In addition to his hypertrophic cardiomyopathy, the patient also had several cardiovascular risk factors, for which he is currently under follow-up.

\section{DISCUSSION}

On ECG, the T wave represents rapid ventricular repolarisation (i.e. phase 3) of the ventricular action potential. During phase 3, there is closure of the calcium channels, while the potassium channels remain open, resulting in rapid loss of positive charge from the cardiomyocytes and restoration of the resting membrane potential. As such, the configuration of the $T$ wave is dependent on the spatial-temporal characteristics of ventricular repolarisation.

In normal adults, the $\mathrm{T}$ wave amplitude is usually upright in all leads, except the aVR, aVL, III and V1 leads, with maximal amplitudes in precordial leads V2 and V3.(4) It is usually asymmetrical with a smooth peak. $T$ wave inversions in the right precordial leads (V1 up to V4) are common in children. In normal adults, $\mathrm{T}$ wave inversions may be present, although rare, in up to lead V3. In addition, $\mathrm{T}$ waves should not exceed $5 \mathrm{~mm}$ in the limb leads and $10 \mathrm{~mm}$ in the precordial leads. Any deviation from this normal pattern can thus provide much information about many disease states. Abnormalities in T waves can present as inverted, biphasic, flattened or tall $\mathrm{T}$ waves. The $\mathrm{T}$ wave is considered inverted if the inversion is deeper than $1.0 \mathrm{~mm}$, and flattened if it is between $1 \mathrm{~mm}$ and $-1.0 \mathrm{~mm} .{ }^{(5)}$ In both the illustrated cases, the identification of an abnormal $\mathrm{T}$ wave provided clues that enabled the cardiac diagnoses to be eventually established.

Causes of $\mathrm{T}$ wave abnormalities are varied and include functional/physiological variants (e.g. postprandial state),

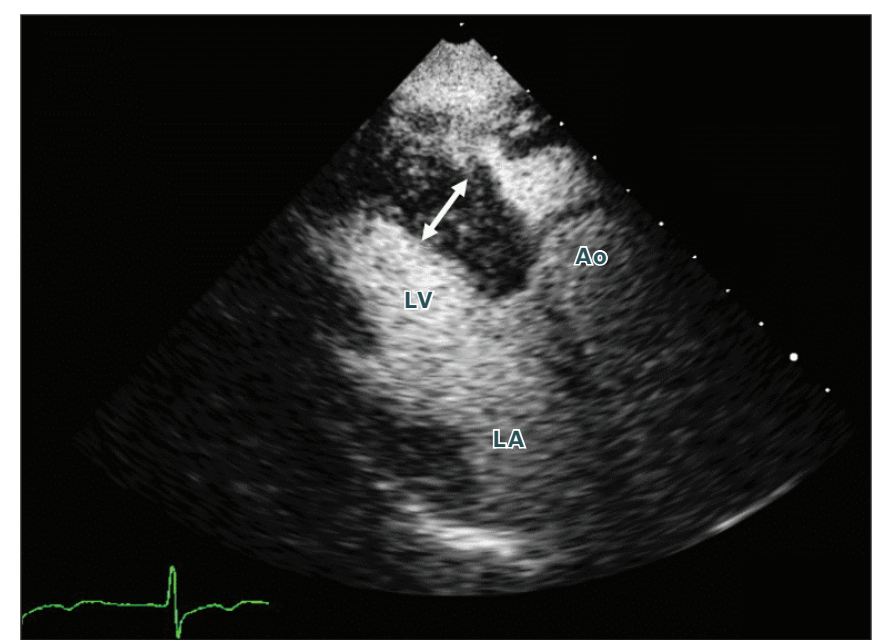

Fig. 4 Contrast echocardiogram in the parasternal long axis view shows asymmetrical ventricular septal wall thickening (white arrows). The septum (indicated by white arrows) measures $19 \mathrm{~mm}$. Ao: aorta; LA: left atrium; LV: left ventricle

endocrine/electrolyte abnormality, myocardial ischaemia, myocarditis/pericarditis/cardiomyopathy, postcardiac surgery state, pulmonary embolism, fever/infection, anaemia, acidosis/ alkalosis, drugs, endogenous catecholamine, metabolic changes, acute abdominal process, intracranial pathology, and systemic disease/amyloidosis/lung diseases. ${ }^{(6)}$

As mentioned earlier, $\mathrm{T}$ wave inversion is commonly seen in normal children, but rarely in normal adults. In a Finnish study of 10,899 asymptomatic middle-aged subjects, T wave inversions in the right precordial leads (i.e. V1 to V3) were seen in 54 subjects and $\mathrm{T}$ wave inversions in leads other than $\mathrm{V} 1$ to $\mathrm{V} 3$ in 76 subjects. After an average follow-up period of 30 years, it was noted that right precordial $\mathrm{T}$ wave inversions did not predict increased mortality, whereas inverted $T$ waves in leads other than V1 to V3 were associated with increased risks of cardiac and arrhythmia deaths. ${ }^{(7)}$

In the two cases discussed in this article, there was either a positive physical finding of a cardiac murmur or a presentation of chest pain. As such, the T wave inversions on their ECG are likely to represent underlying cardiac diseases. For instance, the ECG in Case 1 showed deep T wave inversions, which suggest underlying ischaemic heart disease; together with the clinical scenario, a diagnosis of non-ST-elevation myocardial infarction was made.

Common ECG patterns seen in ischaemic heart disease include: (a) ST segment depression or T wave inversion during myocardial ischaemia directed in the opposite direction as the QRS complex; (b) ST segment depression with upright $\mathrm{T}$ wave; (c) a positive-negative biphasic $\mathrm{T}$ wave pattern; (d) T wave symmetrically inverted with a pointed configuration, while the ST segment is either upwardly bowed (i.e. coved) or horizontally depressed, or not deviated; and (e) ST segment depression progressing to $\mathrm{T}$ wave abnormality during ischaemia free intervals. ${ }^{(4)}$

$\mathrm{T}$ wave changes in patients with severe ischaemia or injury in the distribution of the left anterior descending artery, 
known as Wellens' syndrome, has also been observed by de Zwaan et al. ${ }^{(8)}$ This syndrome is characterised by two patterns of $\mathrm{T}$ wave changes. de Zwaan et al found that in $75 \%$ of the cases, $\mathrm{T}$ waves were symmetrically inverted $>5 \mathrm{~mm}$ from leads V2 to V4, while $25 \%$ of cases had positive-negative $\mathrm{T}$ wave morphology in leads V2 through V4. In both patterns, the ST segments were either isoelectric or minimally deviated $(<1 \mathrm{~mm})$. It also noted that $75 \%$ of the cohort with such $\mathrm{T}$ wave abnormalities, who were treated medically without angiographic investigation, subsequently developed extensive anterior wall myocardial infarction within a mean period of 8.5 days. ${ }^{(8)}$

Another important cause of deep $\mathrm{T}$ wave inversions is hypertrophic cardiomyopathy, a disease characterised by excessive thickening of the left, and occasionally the right, ventricular myocardium. The prevalence of hypertrophic cardiomyopathy is estimated to be 1 in 500 patients. It can be associated with either left ventricular outflow tract obstruction or no significant resting outflow tract gradient (in 75\% of cases). ${ }^{(9)}$ The 12 -lead ECG is abnormal in $75 \%-95 \%$ of patients with hypertrophic cardiomyopathy. Common ECG patterns include evidence of left ventricular hypertrophy, large QRS voltages with giant negative $\mathrm{T}$ waves, as well as deep and narrow Q waves. ${ }^{(10)}$ Giant negative T waves are, however, more characteristic of patients with nonobstructive apical hypertrophic cardiomyopathy. ${ }^{(11)}$

In Case 2, the ECG showed evidence of left ventricular hypertrophy according to both the Sokolow-Lyon and Cornell criteria. ${ }^{(2,3)}$ It also showed widespread ST depressions in the anterolateral precordial leads, representing the 'strain pattern'. Echocardiogram subsequently confirmed the diagnosis of nonobstructive hypertrophic cardiomyopathy. The patient also underwent a contrast echocardiogram, which enabled better delineation of the left ventricular endocardial borders (Fig. 4). The administration of ultrasonography contrast agent is a safe and noninvasive method of enhancing the diagnostic capabilities of echocardiography. In contrast echocardiography, microbubble contrast agents are injected intravenously while echocardiographic images are being acquired. The microbubbles show up as echodense particles within the cardiac chambers and great vessels, allowing for better delineation of cardiac anatomy, ${ }^{(12)}$ as seen in Case 2.

In summary, there are a multitude of causes for $\mathrm{T}$ wave inversions on ECG. Correlation of the ECG with careful history taking and physical examination, together with directed cardiac investigations, will often reveal the underlying cardiac pathology.

\section{ACKNOWLEDGEMENT}

We acknowledge Professor Chia Boon Lock for his advice and help in the writing of this article.

ABSTRACT The causes of abnormal T waves on electrocardiography are multiple and varied. Careful clinical history taking and physical examination are necessary for accurate identification of the cause of such abnormalities. Subsequent targeted specialised cardiac investigations, such as echocardiography or coronary angiography, may be of importance in the diagnosis of the underlying cardiac pathology. We present two cases of $T$ wave inversions with markedly different aetiologies.

Keywords: ECG, hypertrophic cardiomyopathy, ischaemic heart disease, $T$ wave abnormalities

\section{REFERENCES}

1. Poh KK, Teo SG, Tay EL, Yip JW. Electrocardiography Series. ECGs of structural heart disease: Part 1. Singapore Med J 2011; 52:855-8; quiz 859.

2. Sokolow M, Lyon TP. The ventricular complex in left ventricular hypertrophy as obtained by unipolar precordial and limb leads. Am Heart J 1949; 37:161-86.

3. Casale PN, Devereux RB, Alonso DR, Campo E, Kligfield P. Improved sexspecific criteria of left ventricular hypertrophy for clinical and computer interpretation of electrocardiograms: validation with autopsy findings. Circulation 1987; 75:565-72.

4. Hanna EB, Glancy DL. ST-segment depression and T-wave inversion: classification, differential diagnosis, and caveats. Cleve Clin J Med 2011; 78:404-14.

5. Rautahraju PM, Surawicz B, Gettes LS, et al. AHA/ ACCF/ HRS recommendations for the standardization and interpretation of the electrocardiogram: part IV: the ST segment, T and $U$ waves, and the QT interval: a scientific statement from the American Heart Association Electrocardiography and Arrhythmias Committee, Council on Clinical Cardiology; the American College of Cardiology Foundation; and the Heart Rhythm Society. Endorsed by the International Society for Computerized Electrocardiography. J Am Coll Cardiol 2009; 53:982-91.

6. Prutkin JM. ECG tutorial: ST and T wave changes. In: UpToDate Inc [online]. Available at: http://www.uptodate.com/contents/ecg-tutorialst-and-t-wave-changes. Accessed October 14, 2013.

7. Aro AL, Anttonen O, Tikkanen JT, et al. Prevalence and prognostic significance of T-wave inversions in right precordial leads of a 12lead electrocardiogram in the middle-aged subjects. Circulation 2012; 125:2572-7.

8. de Zwaan C, Bär FW, Wellens HJ. Characteristic electrocardiographic pattern indicating a critical stenosis high in left anterior descending coronary artery in patients admitted because of impending myocardial infarction. Am Heart J 1982; 103(4 Pt 2):730-6.

9. Maron BJ. Hypertrophic cardiomyopathy: a systematic review. JAMA 2002; 287:1308-20.

10. Prinz C, Farr M, Hering D, Horstkotte D, Faber L. The diagnosis and treatment of hypertrophic cardiomyopathy. Dtsch Arztebl Int 2011; 108:209-15.

11. Yamaguchi $\mathrm{H}$, Ishimura $\mathrm{T}$, Nishiyama $\mathrm{S}$, et al. Hypertrophic nonobstructive cardiomyopathy with giant negative $\mathrm{T}$ waves (apical hypertrophy): ventriculographic and echocardiographic features in 30 patients. Am J Cardiol 1979; 44:401-12.

12. Mulvagh SL, Rakowski H, Vannan MA, et al. American Society of Echocardiography Consensus Statement on the Clinical Applications of Ultrasonic Contrast Agents in Echocardiography. J Am Soc Echocardiogr 2008; 21:1179-201. 


\section{SINGAPORE MEDICAL COUNCIL CATEGORY 3B CME PROGRAMME} (Code SMJ 201311A)

Question 1. In the interpretation of T waves on an electrocardiogram (ECG):

(a) $T$ wave represents rapid ventricular depolarisation.

(b) T wave amplitude should not exceed $5 \mathrm{~mm}$ in the limb leads.

(c) T wave should not exceed $15 \mathrm{~mm}$ in the precordial leads.

(d) T wave is usually upright in lead aVR.

Question 2. T wave abnormalities on an ECG can be caused by:
(a) Acute myocardial infarction.
(b) Recent cardiac surgery.
(c) Acute intracranial haemorrhage.
(d) Severe sepsis with metabolic acidosis.

Question 3. Regarding T wave inversions in ischaemic heart disease:

(a) T inversions are always seen with depressed ST segments.

(b) Inverted T waves are usually symmetrical with pointed configuration.

(c) Wellens' syndrome describes $T$ wave changes in the precordial leads associated with myocardial ischaemia in the left anterior descending artery territory.

(d) T wave inversions can only be seen during acute ischaemic periods.

Question 4. Regarding hypertrophic cardiomyopathy:

(a) It can be classified by the presence or absence of right ventricular outflow tract obstruction.

(b) ECG is normal in the majority of patients.

(c) Left ventricular hypertrophy pattern can be seen on ECG.

(d) Large QRS complexes and giant negative T waves can be seen on ECG.

Question 5. Regarding the approach to patients with significant T wave inversions on ECG:

(a) To diagnose any underlying cardiac pathology, thorough history taking and physical examination is key.

(b) Family history of sudden cardiac death is significant.

(c) T wave inversion in the precordial leads is common in prepubertal children.

(d) Right precordial leads (V1 to V3) T wave inversions on ECG in asymptomatic adults have been shown to be associated with higher cardiovascular mortality.

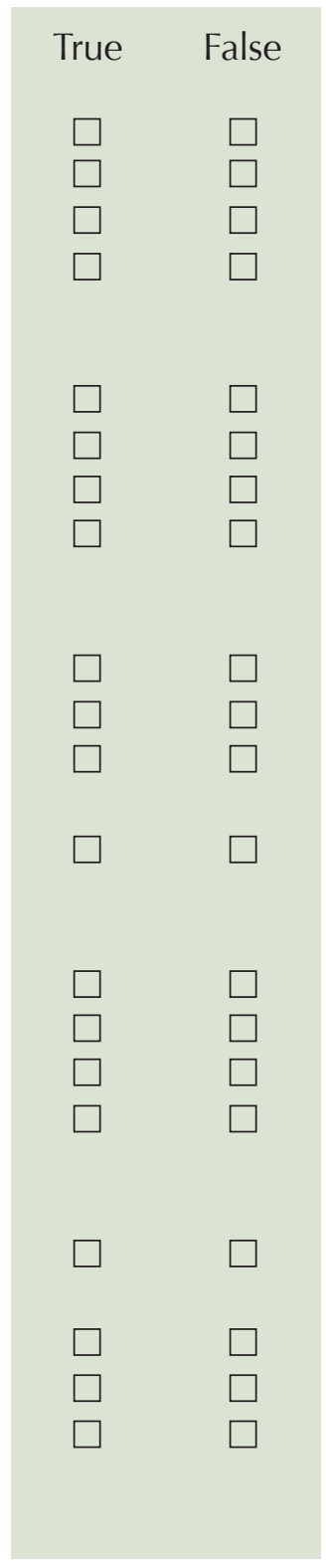

\section{Doctor's particulars:}

Name in full

MCR number

Email address 